\section{AURICULAR FIBRILLATION}

\section{WITH SPECIAL REFERENCE TO RHEUMATIC HEART DISEASE}

BY

\section{H. R. L. FRASER, M.B., M.R.C.P.Ed.}

AND

\section{R. W. D. TURNER, O.B.E., M.D., F.R.C.P. F.R.C.P.Ed.}

(From the Department of Medicine, University of Edinburgh, and the Cardiac Department, Western General Hospital, Edinburgh)

"... The auricles pipe and the ventricles perforce must dance . . . a dance that sometimes leads to death"

(Hay and Jones, 1927).

\section{Aetiology}

The most common causes of auricular fibrillation are rheumatic heart disease and degenerative heart disease due to coronary atheroma and hypertension. Less common causes are thyrotoxicosis and constrictive pericarditis. Rare causes include pulmonary embolism, acute respiratory infections, carcinoma involving the mediastinum, accidental electric shock, electric convulsion therapy, and the administration of digitalis. In addition it seems clear that auricular fibrillation may sometimes occur spontaneously, and recur or persist over many years without evidence of underlying organic heart disease. Fibrillation may follow thoracotomy in older patients without clinical evidence of underlying heart disease.

The highest incidence in the general population is probably from degenerative heart disease, but this is not necessarily reflected in hospital statistics, because the elderly are more commonly treated at home by their doctors than referred to hospital.

The precise relationship of auricular fibrillation to hypertensive heart disease and to coronary atheroma is obscure. Although in hypertension the coronary arteries may be remarkably free from atheroma the two conditions are often associated, as pointed out by Harrison and Wood (1949). These authors consider that hypertension is more important than coronary atheroma in the genesis of auricular fibrillation, but in our experience most patients who die from cardiac failure secondary to hypertension do so while still in sinus rhythm. All that can be said is that auricular fibrillation commonly accompanies cardiac failure in middle-aged and elderly persons in whom the only demonstrable pathological abnormalities are left ventricular hypertrophy and/or coronary atheroma.

Sinus rhythm is the rule previous to the onset of angina pectoris or of myocardial infarction, although fibrillation may follow infarction. The rarity of auricular fibrillation in syphilitic heart disease is well known, and is presumably due to the absence of involvement of the myocardium by this infection. Auricular fibrillation due to thyrotoxicosis is now far less common owing to successful medical and surgical management of the disease in the early stages, but it is still seen in middleaged patients, particularly when the more obvious manifestations of thyrotoxicosis are not florid. Sometimes it is the presenting sign. Auricular fibrillation secondary to the administration of digitalis was referred to by
Mackenzie in 1911 and is well recognized. Apart from its occurrence in adults it has been noted in children being treated for rheumatic heart disease. This association serves to emphasize that digitalis should never be given without good reason.

Benign auricular fibrillation is by no means uncommon. In our experience auricular fibrillation in healthy adults may apparently be related to overwork, oversmoking, and emotion. Normal rhythm can usually be restored with quinidine, but if paroxysms tend to recur it is far better to give a maintenance dose of digitalis instead, because paroxysmal attacks are usually associated with unpleasant sensations from tachycardia and palpitation.

\section{Auricular Fibrillation in Rheumatic Heart Disease}

Of 500 consecutive hospital patients in whom auricular fibrillation was recorded electrocardiographically, $30 \%$ had rheumatic heart disease. A study has been made of 500 patients with mitral disease over a period of five years: 250 were treated surgically, and $42 \%$ of them had auricular fibrillation before operation. The other 250 were treated medically over approximately the same period of time-115 were regarded as unsuitable for operation because the condition was too mild, and 135 because it was too severe or because some other factor complicating mitral stenosis was predominant-for example, mitral incompetence, aortic valvular disease, systemic hypertension, or pulmonary disease. Auricular fibrillation occurred in $80 \%$ of the severe group, and in $4 \%$ of the mild group.

Precipitating Factors.-Among the factors we have observed are exercise, emotion, infection, and, we believe, a flare-up of carditis, but frequently there is no obvious cause, the patient being unaware of any change in his symptoms. Exercise and emotion may both be associated with increased sympathetic activity, or possibly the association of mitral valve disease and an attempt to increase the cardiac output may result in atrial dilatation and mechanical stimulation.

A woman of 47 who regularly attended the clinic and was known to be in sinus rhythm was looking out of the window of her room and saw a child run over by a car. Immediately she experienced severe palpitation and breathlessness, and was sent by her practitioner to hospital, where auricular fibrillation was discovered. Normal rhythm was readily restored with quinidine.

The role of active carditis is considered below in the discussion on aetiology.

\section{Disadvantages of Auricular Fibrillation}

The onset of auricular fibrillation may have unpleasant or serious consequences or may occur without any untoward incident. Frequently the patient is unaware of the change in rhythm. Symptoms are dependent on the severity of the underlying heart disease and the effect of tachycardia, the increase in heart rate being the immediate precipitating factor.

Dyspnoea may be extreme and pulmonary oedema may soon develop if the ventricular rate is not controlled by digitalis. Palpitation may be attributed to the rapid heart rate and the irregularity which is responsible for beats of varying force. Anxiety is readily understood and requires no amplification. Syncope may be due to the fall in cardiac output which follows the onset of fibrillation. However, it has been shown that the change of rhythm is often accompanied by a period of ventricular arrest of sufficient duration to. cause cerebral ischaemia and loss of consciousness (Comeau, 1942). In three of our patients this explanation seemed probable in that tachycardia was not severe. It is noteworthy that syncope may follow the cessation of a paroxysm. Retrosternal oppression is common and may be 
associated with the intense dyspnoea at the onset of the arrhythmia, or possibly, in patients with tight mitral stenosis, be due to myocardial ischaemia. Cardiac failure is dependent on uncontrolled tachycardia and may be very severe, with intense dyspnoea, engorgement of veins and liver, and, later, peripheral oedema.

A woman of 38 had a considerable disability from tight mitral stenosis with sinus rhythm, but had never experienced pulmonary oedema or congestive failure. Her name was placed on the waiting-list for operation, but she was not given priority. A few weeks later she was admitted as an emergency, having wakened during the night with alarming intense palpitation. She became very breathless and dizzy, broke out in a cold sweat, and was sick. Her doctor was called, found she had fibrillation, with a rapid ventricular rate, and gave digitalis, but not long after she developed pulmonary infarction. On arrival she was in severe congestive failure and jaundiced either from the gross engorgement of the liver or from pulmonary infarction. She was subsequently subjected to mitral valvulotomy and has remained well for the past two years.

Pulmonary infarction may follow the onset of auricular fibrillation almost immediately, and may be attributable to: (1) stasis with thrombus formation in the right auricle or atrium and subsequent embolism; (2) local thrombosis in a branch of the pulmonary artery in association with pulmonary congestion; (3) thrombosis in a peripheral vein in association with slowing of the circulation and subsequent pulmonary embolism. Systemic embolism may likewise be attributable to thrombosis in the left auricle or atrium from stasis behind a stenosed mitral valve or in association with mural endocarditis. Decreased capacity for effort may possibly depend on relative inability to increase cardiac output on exertion, as shown by Blumgart (1924), but it may sometimes be due to tachycardia insufficiently controlled by digitalis.

Probability of Congestive Failure During Pregnancy.Patients with auricular fibrillation are customarily advised not to become pregnant, or, if pregnant, to have the pregnancy terminated on account of the frequency of cardiac failure in the later months. It is difficult to say to what extent the cardiac failure is attributable to the auricular fibrillation itself or to the severity of the underlying heart disease which fibrillation portends.

\section{Auricular Fibrillation and Mitral Valvulotomy}

Auricular fibrillation is a disadvantage, but is not in itself a contraindication to mitral valvulotomy. The disadvantages are from the presumed presence of considerable myocardial damage, and from the increased probability of clot being present in the auricle or atrium. These factors are discussed fully below. Auricular fibrillation had occurred pre-operatively in $30 \%$ of this series. In addition, $56(23 \%)$ of those previously in sinus rhythm developed auricular fibrillation in the immediate post-operative period. So far only four patients have developed auricular fibrillation subsequently.

Prognosis as regards life is less favourable than in those with sinus rhythm (see below).

\section{Relative Advantages of Established Auricular Fibrillation}

Although, on balance, auricular fibrillation must be considered a disadvantage, it has relative advantages: (1) Control of heart rate: in the presence of organic heart disease tachycardia may be serious. Exercise, emotion, or infection may cause unpleasant symptoms or precipitate cardiac failure or intracardiac thrombosis. There is no way of controlling sinus tachycardia, but with auricular fibrillation the ventricular rate can usually be reduced or a rise in rate prevented in some degree by the maintenance of full digitalization. Tachycardia may be a serious disadvantage in specialized examinations such as cardiac catheterization or during or following operation. (2) Many authors have referred to the relative infrequency with which patients with auricular fibrillation develop subacute bacterial endo- carditis. (3) Freedom from paroxysmal attacks: the disadvantages of paroxysmal auricular fibrillation have already been discussed.

\section{Pathogenesis}

\section{Auricular Fibrillation and the Degree of Mitral Stenosis}

Table I shows the relationship of auricular fibrillation to the degree of stenosis. Of 207 patients with severe stenosis, 90 had auricular fibrillation and 117 sinus rhythm. Of 37 with moderately severe stenosis, 12 had auricular fibrillation and 25 had sinus rhythm. Since almost all patients in this operative series had severe or moderately severe stenosis, the findings in a parallel series who were operated upon for predominant mitral incompetence have been added. Of a total series of 25 patients with some degree of mitral stenosis but predominant mitral incompetence, 14 had auricular fibrillation and 11 sinus rhythm.

TABle I.-Cardiac Rhythm and Degree of Mitral Stenosis

\begin{tabular}{llll|c|c}
\hline \multicolumn{2}{c|}{ Degree of } & Mitral Stenosis & Sinus Rhythm & Auricular Fibrillation \\
\hline $\begin{array}{llll}\text { Severe } \\
\text { Moderate }\end{array}$ &. &. &. & 117 & 90 \\
Mild &. &. &. & 25 & 12 \\
\hline & Total &. &. & 14 & 11 \\
\hline
\end{tabular}

We have also examined this relationship for the 250 patients who were not subjected to surgical treatment. From an analysis of all this material it is concluded that auricular fibrillation bears no direct relationship to the degree of stenosis or incompetence of the mitral valve.

Auricular Fibrillation and Pulmonary Hypertension

Table II shows the relationship of auricular fibrillation to pulmonary hypertension in 100 consecutive patients in whom cardiac catheterization was carried out. In most instances resistances were not calculated, but it is considered

TABLE II.-Cardiac Rhythm and Degree of Pulmonary Hypertension

\begin{tabular}{|c|c|c|c|c|}
\hline \multirow{2}{*}{$\begin{array}{l}\text { Pulmonary } \\
\text { Hypertension } \\
\text { Grade }\end{array}$} & \multicolumn{2}{|c|}{ Sinus Rhythm } & \multicolumn{2}{|c|}{ Auricular Fibrillation } \\
\hline & No. & $\%$ & No. & $\%$ \\
\hline $\begin{array}{l}1 \\
2 \\
3\end{array}$ & $\begin{array}{l}15 \\
22 \\
24\end{array}$ & $\begin{array}{l}24 \\
36 \\
38\end{array}$ & $\begin{array}{r}9 \\
16 \\
13\end{array}$ & $\begin{array}{l}24 \\
42 \\
34\end{array}$ \\
\hline Total & 61 & & 39 & \\
\hline
\end{tabular}

that the resting pressures with the patients relaxed and pressures after exercise give a fair indication of the degree of pulmonary hypertension present. For the purpose of this comparison the patients were graded into three degrees of severity: (1) slight $=$ mean pulmonary-artery pressure $<35$ $\mathrm{mm}$. $\mathrm{Hg}$, (2) moderate = mean P.A. pressure of $36-50 \mathrm{~mm}$. $\mathrm{Hg}$, (3) severe $=$ mean P.A. pressure of $50+\mathrm{mm}$. Hg. In borderline cases the response to exercise was considered in the grading. It is evident that there is no relationship between cardiac rhythm and the degree of pulmonary hypertension (Fraser and Turner, 1955).

\section{Cardiac Enlargement}

It has been shown that auricular fibrillation cannot be correlated with such mechanical and haemodynamic factors as the degree and duration of mitral stenosis or incompetence and pulmonary hypertension. The association with cardiac enlargement is more close.

Table III shows the relationship of fibrillation to cardiac enlargement. Of $\mathbf{4 8}$ patients in whom there was little if any enlargement (cardiothoracic ratio $<50 \%$ ), 43 had sinus rhythm and 5 auricular fibrillation. Of 51 with considerable enlargement (cardiothoracic ratio $>60 \%$ ), 13 had sinus rhythm and 38 fibrillation. Of the 101 with moderate enlargement the incidence of sinus rhythm and fibrillation was about equal. 
TABLE III.-Cardiac Rhythm and Cardiac Enlargement

\begin{tabular}{|c|c|c|c|c|c|c|c|c|c|c|c|c|c|c|}
\hline \multirow{2}{*}{$\begin{array}{l}\text { Cardio- } \\
\text { thoracic } \\
\text { Ratio \% }\end{array}$} & \multicolumn{2}{|c|}{ Sinus Rhythm } & \multicolumn{2}{|c|}{ Auric. Fib. } & \multirow{2}{*}{$\begin{array}{c}\text { Degree of } \\
\text { Left Atrial } \\
\text { Enlargement }\end{array}$} & \multicolumn{2}{|c|}{ Sinus Rhythm } & \multicolumn{2}{|c|}{ Auric. Fib. } & \multirow{2}{*}{$\begin{array}{c}\text { Degree of } \\
\text { Right Atrial } \\
\text { Enlargement }\end{array}$} & \multicolumn{2}{|c|}{ Sinus Rhythm } & \multicolumn{2}{|c|}{ Auric. Fib. } \\
\hline & No. & $\%$ & No. & $\%$ & & No. & $\%$ & No. & $\%$ & & No. & $\%$ & No. & $\%$ \\
\hline $\begin{array}{l}<50 \\
50-54 \\
55-59 \\
60-72\end{array}$ & $\begin{array}{l}43 \\
23 \\
36 \\
13\end{array}$ & $\begin{array}{l}38 \\
20 \\
31 \\
11\end{array}$ & $\begin{array}{r}5 \\
14 \\
28 \\
38\end{array}$ & $\begin{array}{r}6 \\
16 \\
33 \\
45\end{array}$ & $\begin{array}{c}1 \\
2 \\
3 \\
4 \\
\text { Aneurysmal }\end{array}$ & $\begin{array}{r}64 \\
41 \\
8 \\
2 \\
0\end{array}$ & $\begin{array}{r}55 \\
35 \\
7 \\
2 \\
-\end{array}$ & $\begin{array}{r}19 \\
36 \\
19 \\
7 \\
4\end{array}$ & $\begin{array}{r}22 \\
42 \\
22 \\
8 \\
5\end{array}$ & $\begin{array}{l}0 \\
1 \\
2 \\
3 \\
4\end{array}$ & $\begin{array}{r}60 \\
34 \\
18 \\
3 \\
0\end{array}$ & $\begin{array}{r}52 \\
29 \\
16 \\
3 \\
-\end{array}$ & $\begin{array}{r}18 \\
19 \\
28 \\
19 \\
1\end{array}$ & $\begin{array}{r}21 \\
22 \\
33 \\
22 \\
1\end{array}$ \\
\hline
\end{tabular}

Left Atrium.-Of 83 patients with slight atrial enlargement, 64 had sinus rhythm and 19 fibrillation; of 40 with considerable enlargement, 10 had sinus rhythm and 30 fibrillation; and of 77 with moderate enlargement the incidence of sinus rhythm and fibrillation was about equal.

Right Atrium.-Of 78 patients without right atrial enlargement 60 had sinus rhythm and 18 fibrillation; of 23 with considerable enlargement, 3 had sinus rhythm and 20 fibrillation; and of 99 with slight or moderate enlargement the incidence of sinus rhythm and fibrillation was about equal.

It can be seen that those with fibrillation tend to have larger hearts than those with sinus rhythm. This might be interpreted as indicating either that fibrillation resulted in cardiac enlargement or that the enlargement and the fibrillation both resulted from the same cause. We consider the latter to be the more probable explanation.

Auricular fibrillation is not necessarily associated with much atrial enlargement, because in this series there were $22 \%$ with only slight left atrial enlargement and $21 \%$ without any apparent right atrial enlargement.

Contrary to the general belief, our experience indicates that, apart from resultant cardiac failure, the onset of fibrillation does not usually result in an increase in the cardiothoracic ratio or in the size of the right or the left atrium. Although the heart is often much enlarged when the patient is first seen, cardiac failure apart, it has been uncommon for progressive enlargement to be observed in patients who were kept under observation but not subjected to operation.

We have been unable to correlate heart size with age, the number of attacks of rheumatic fever, the number of years since the first attack, or the degree of mitral stenosis or incompetence. Presumably it is a direct effect of the rheumatic process on the myocardium which is responsible for cardiac enlargement.

\section{Histology}

In children and adolescents the onset of auricular fibrillation is often associated with rheumatic activity. This is illustrated in our non-operative series in a girl of 17 who was rejected for surgical treatment. She developed fibrillation six months before death from cardiac failure. At necropsy there was heavy infiltration of the myocardium with Aschoff nodules. In older people fibrillation is probably more often due to the long-continued effects of a damaged myocardium, but a surprisingly high proportion of biopsies indicating active myocarditis has .been obtained in those over 40.

In the surgical series there were seven patients under the age of 30 who had auricular fibrillation prior to operation. Histological evidence of rheumatic activity was present in four of those in whom biopsies were taken. There were eight patients under the age of 30 , previously in sinus rhythm, who developed fibrillation in the early postoperative period, and the biopsies were positive in six of the seven in whom they were taken. On the other hand, positive biopsies were also found in 20 out of 40 patients under the age of 30 in sinus rhythm.

The difficulty in identifying activity of the rheumatic process is well recognized, and the correlation between clinical, haematological, electrocardiographic, and histological findings is poor. There may be differences of opinion between pathologists concerning the significance of aggregations of lymphocytes, plasma cells, and histiocytes, but the Aschoff nodule is generally accepted as the hall-mark of active rheu- matism. We have had many cases with Aschoff nodules from the left auricular appendix in the absence of any other supporting evidence of rheumatic activity. We have also had patients who have had to be operated upon despite suggestive evidence of mildly active rheumatism, but in whom the biopsy was negative, and others, not in this series, who have died with clinical evidence of active carditis without histological support of the diagnosis. It seems clear that present histological methods do not always reveal important degrees of carditis.

No direct proof of the pathogenesis of auricular fibrillation can be obtained. By a process of exclusion it seems clear that factors other than that of myocardial damage are unimportant, and there is presumptive evidence that the latter factor is responsible although its nature is obscure.

\section{Auricular Fibrillation and Intracardiac Thrombosis}

In this series of 250 cases auricular fibrillation occurred pre-operatively in $106(42 \%)$, and in $43(40 \%)$ of these 106 cases clot in the left auricle or atrium was found at operation. In addition, in a few cases in which it had not been suspected by the surgeon, evidence of pre-operative thrombosis was found in the biopsy of left aur.cle material taken at operation.

Clot was found in only 3 of the 144 patients in sinus rhythm ( $2 \%)$. Hence, as widely recognized for many years, whatever other factors may be operative, it is clear that auricular fibrillation unquestionably predisposes to thrombus formation.

We are not here concerned with other possible aetiological factors such as roughening of the endocardial lining from the effects of rheumatic carditis or an increase in the clotting tendency of the blood, but stasis is important and clearly the more severe the degree of mitral stenosis the greater the degree of stasis behind the narrowed valve. The presence of congestive cardiac failure will have a similar effect, especially in connexion with auricular fibrillation.

An analysis of our material shows that clot occurred most frequently with severe stenosis, less commonly with moderately severe stenosis, and rarely with mild stenosis. The presence of a regurgitant jet seems to militate against thrombus formation, as might be expected.

We have met only one instance of thrombus formation in a patient in whom the evidence suggested that the stenosis was mild and there was no incompetence. This occurred in a man in whom it presumably developed following the onset of auricular fibrillation. He was seen next day and normal rhythm was readily restored with quinidine. but unfortunately this was followed by systemic embolism. This occurred early in our experience and before we had developed our present policy of not making any attempt to restore normal rhythm soon after its onset, particularly if the patient is likely to be subjected to operation, when the opportunity will be provided of emptying the auricle or atrium of clot before carrying out valvulotomy. In this case no other symptoms relevant to mitral stenosis were present and there was little cardiac enlargement and no evidence of pulmonary hypertension or right ventricular hypertrophy. Consequently operation was not advised. It would have been better at least to have waited for some months until it could be assumed that the clot was firmly adherent. Presumably in cases such as this mural endocarditis is the important factor. 
In this series all 32 patients who had experienced systemic embolism pre-operatively had auricular fibrillation, and 22 $(69 \%)$ of them were found to have clot in the auricle or atrium at operation. Of these 22 patients, 5 sustained operative embolism. A past history of systemic embolism must therefore be taken to indicate an additional increased hazard from the operative procedure-that is, the disturbance of clot by the surgeon's finger. On the other hand, it is likely that a patient who has experienced embolism from intracardiac thrombosis secondary to rheumatic heart disease will experience another such episode in due course, and there is good reason to believe that a reduction in the degree of mitral stenosis by successful valvulotomy decreases the probability of further thrombus formation in the future. So far not one of the 32 patients with a past history of systemic embolism has experienced further embolism in the years following operation.

The question of right-sided thrombus formation is more difficult to analyse except in post-mortem material. In the first place the right auricle and atrium are not explored at operation and so precise information cannot be obtained during life. Also, whereas for practical purposes the left auricle and atrium are the only sources of systemic embolism, there are other sources of pulmonary infarction than embolism from the right side of the heart. Thrombosis may occur in a main branch of the pulmonary artery or in one of its subdivisions, and pulmonary embolism may result from thrombosis in a peripheral systemic vein. However, it is evident that, for whatever reason in the individual case, auricular fibrillation also predisposes to pulmonary embolism and infarction.

\section{Prognosis of Auricular Fibrillation in Rheumatic Heart Disease}

According to Degraff and Lingg (1935) and to Stroud et al. (1932), the average duration of life following the permanent establishment of auricular fibrillation after the age of 30 in persons with rheumatic heart disease is two years, and less than one year when fibrillation begins before the age of 20 . Put another way, only $25 \%$ will live three years or more after the onset of the arrhythmia. Stroud et al. found that the average age of onset was 39 , that $86 \%$ of 65 patients developed auricular fibrillation before 40 , and that $88 \%$ had died before the age of 53. It is probable that with the introduction of the antibiotics and the more widespread use of mercurial diuretics the prognosis has been improved, but long before the days of surgical treatment for mitral stenosis it was apparent that the prognosis of those with auricular fibrillation was worse than those with sinus rhythm. It is only to be expected that the prognosis in these patients after operation is also likely to be not so good as with those in sinus rhythm. Actually it is not possible to analyse the influence of auricular fibrillation per se in relation to the operative results because of the other variable factors involved. Those with fibrillation tend to be older, to have larger hearts, and to have intracardiac thrombosis more often than those in sinus rhythm; also, they more frequently have a past history of congestive failure. Nevertheless it is clear that very worthwhile results may be obtained in those with fibrillation. Apart from the direct benefit of the valvulotomy itself, if, as we believe, fibrillation is often a reflection of myocardial damage, then it is reasonable to suppose that benefit will accrue from the damaged muscle having to work against less resistance.

In this series congestive failure occurred more frequently in those with auricular fibrillation than in those with sinus rhythm, but it is often difficult from the history to decide whether or not a patient has had congestive failure. In untreated patients oedema from failure is usually if not always accompanied by raised venous pressure or at least a positive hepato-jugular reflux. Middle-aged women, especially, often complain of intermittent swelling of the ankles towards the end of the day, and a certain diagnosis may be impossible unless the patient is examined when the oedema is present.
It is too soon to be able to assess the effects of valvulotomy in prolonging life in those with auricular fibrillation, but from the initial benefit which has followed operation in many cases it seems probable that life will in fact be materially prolonged.

\section{Post-operative Auricular Fibrillation}

Post-operative auricular fibrillation in those previously in sinus rhythm is of common occurrence and developed in $23 \%$ of the patients in this series. This incidence agrees well with the experience of other observers. In no instance has fibrillation developed during the actual operation despite the fact that nodal rhythm, wandering pace-maker, multifocal extrasystoles, and runs of ventricular tachycardia commonly occur. Nor has auricular fibrillation developed on the day of the operation, but at various intervals thereafter over the next ten days. On occasion the onset has coincided with some minor surgical procedure such as paracentesis thoracis or bronchoscopy, but usually there has been no obvious reason, and often it developed in relatively young patients and in those otherwise apparently undergoing a smooth convalescence. Except in the older age groups, auricular fibrillation is rare after thoracotomy for procedures not involving opening the pericardium-for example, for pulmonary resection.

Presumably there are two relevant factors in patients undergoing valvulotomy - that of rheumatic heart disease, and the traumatic pericarditis which occurs in varying degree in all cases. The pericardial reaction as judged by fever, pain, and rub varies greatly from patient to patient, and we have not found any relationship between its apparent severity and the incidence of post-operative fibrillation. Nevertheless, if the characteristic electrocardiographic changes of pericarditis are in fact due to inflammation of the underlying epicardium, then the striking pattern present after valvulotomy implies a likely stimulus to auricular fibrillation.

From our experience we have been unable to decide whether the pre-operative administration of dig talis and its continuance during and following operation in those with sinus rhythm affects the incidence of post-operative fibrillation. Some observers consider that digitalis should not be given because it actually increases the likelihood of fibrillation. Theoretically, previous digitalization would be expected at least to prevent an undue rise in the ventricular rate following the onset of fibrillation.

\section{Restoration of Normal Rhythm}

We found it unprofitable to attempt the restoration of normal rhythm with quinidine in the first ten post-operative days because most patients were resistant during this period, presumably from persistence of the underlying stimulus to fibrillation. However, after the first fortnight normal rhythm has been successfully restored in all but 3 of the 60 patients. With one possible exception there have been no untoward incidents from this procedure. The possible exception occurred in a woman in whom normal rhythm had been restored, and who died suddenly and unexpectedly the day after discontinuance of the drug. Postmortem examination revealed no cause for death, which was probably due to some ventricular arrhythmia, but under the circumstances it is difficult to incriminate quinidine, since the blood level would have been low at the time death occurred.

Prophylactic quinidine given before, during, and after the operation, even in high dosage, was often ineffective in preventing the occurrence of auricular fibrillation.

\section{Summary and Conclusions}

Over a period of five years a study has been made of auricular fibrillation in relation to 500 patients with rheumatic mitral disease. Of these, 250 were treated surgically. 
Factors which may precipitate auricular fibrillation include exercise, emotion, infection, and active carditis, but usually no cause is apparent.

The onset of auricular fibrillation may pass unnoticed or be responsible for dyspnoea, pulmonary oedema, pulmonary infarction, systemic embolism, cardiac failure, loss of consciousness, or anxiety.

Relative advantages of established auricular fibrillation include freedom from paroxysmal attacks, ease of control of heart rate with digitalis, and comparative freedom from bacterial endocarditis.

Persons with auricular fibrillation tend to have larger hearts than those in sinus rhythm, but reasons are given for foncluding that enlargement is not directly related to the arrhythmia.

Possible factors in the pathogenesis of auricular fibrillation are reviewed, and it is concluded that there is presumptive evidence that myocardial damage may be the most important.

Auricular fibrillation is not a contraindication to valvulotomy but a disadvantage from the presumed presence of myocardial damage and the increased probability of clot being present, with the consequent risk of embolism.

Auricular fibrillation had occurred pre-operatively in $42 \%$ of the surgical series. In these 106 patients clot was found in the left auricle or atrium in $40 \%$, but in only 3 of 144 patients in sinus rhythm.

The presence of a regurgitant jet seems to militate against thrombus formation.

Good operative results may be obtained in cases of auricular fibrillation and life thereby prolonged, but the operative risk is greater and the ultimate prognosis poorer than in cases with sinus rhythm.

Post-operative auricular fibrillation occurred in $23 \%$ of those previously in sinus rhythm. In the first 10 post-operative days it is rarely possible to restore sinus rhythm with quinidine, but thereafter success is almost always obtained. Pre-operative prophylactic quinidine is ineffective.

\section{REFERENCES}

Blumgart, H. L. (1924). Heart, 11, 49.

Comeau, W. J. (1942). New Engl. J. Med., 227, 134.

Degraff, A. C., and Lingg, C. (1935). Amer. Heart J., 10, 630

Fraser, H. R. L., and Turner, R. W. D. (1955). Brit. Heart J., 17, 459. Harrison, C. V., and Wood, P. (1949). Ibid.. 11, 205.

Hay, J., and Jones, H. W. (1927). British Medical Journal, 1, 559

Mackenzie, J. (1911). Heart, 2, 273.

Stroud, W. D., Laplace, L. B., and Reisinger, J. A. (1932). Amer. J. med. Sci., 183, 48 .

Among organizations which help to maintain friendly international relations on the medical plane, the Horse Shoe Club is particularly concerned with fostering friendship between American, Canadian, and English men and women interested in the cure and prevention of disease. The club encourages exchange research and clinical appointments between these countries, and provides hospitality for overseas visitors. On November 12, at Apothecaries' Hall, the Horse Shoe Club held its autumn cocktail party (one of two held every year) to entertain doctors from the U.S.A. and Canada who are working here for the time being either in their country's armed Forces or in civilian posts, together with their wives. Dr. Geoffrey Bourne, chairman of the club, Sir Harry Hague, treasurer, and Dr. Claude Nicol, secretary, were all present. Many of the 150 guests took the opportunity of hearing Dr. Neville FinZI, master of the Society of Apothecaries, discourse on some of the treasures of the Society.

\section{ACTINOMYCIN C IN TREATMENT OF ADVANCED HODGKIN'S DISEASE}

BY

\section{J. R. TROUNCE, M.D., M.R.C.P.}

\section{A. B. WAYTE, M.B., D.M.R.E.}

AND

J. M. ROBSON, M.D., D.Sc., F.R.S.Ed.

From the Departments of Medicine, Radiotherapy, and Pharmacology, Guy's Hospital

In 1949 Brockman and Grubhofer isolated from Streptomyces chrysomallis a substance which they named actinomycin C ("sanamycin"). Three years later, Hackmann (1952) reported that this substance had an inhibiting effect on the growth of the Ehrlich carcinoma, a mouse sarcoma, and the Walker, carcinoma in rats. He also noted that actinomycin $\mathrm{C}$ had a cytostatic effect, particularly on the lymphatic system (Hackmann, 1953).

As a result of these findings, Schulte (1952) treated a number of patients with tumours of the reticuloendothelial system with this substance and reported some improvement in their condition, with disappearance of the tumours in some cases and in others a reduction of the dose of radiation required to control.

A large series of patients was investigated by a group of French workers (Presse méd., 1954). It was stated that the effect of actinomycin C in Hodgkin's disease was inconstant and of short duration. Two of the group, however (Ravina and Pestel, 1954), reported considerable improvement in Hodgkin's dišease. Their better results may have been due to the large dosage of actinomycin $\mathrm{C}$, which was given in gradually increasing doses up to $1,200 \mu \mathrm{g}$. a day. Each daily dose was given by intravenous drip spread over three to four hours.

The toxic effects were confined to the gastro-intestinal tract, and consisted of stomatitis, pharyngitis, nausea, and diarrhoea. Actinomycin $\mathrm{C}$ was also tried in acute leukaemia and various carcinomas without effect.

\section{Present Investigation}

Actinomycin $\mathrm{C}$ was given to six patients with Hodgkin's disease and one with a reticulum-celled sarcoma. All the patients had already been treated by radiotherapy, and all but one had received one or more courses of nitrogen mustard; these courses, however, were not completed in two cases owing to toxic effects. All the patients were suffering from an advanced stage of the disease.

Dosage.-The usual scheme of dosage employed was to start with $100 \mu \mathrm{g}$. of actinomycin C intravenously. If no toxic effects were observed the dose was increased so that at the end of a week or ten days the patient was receiving a daily dose of $400 \mu \mathrm{g}$. The dose was then progressively increased to $600 \mu \mathrm{g}$., and finally $800 \mu \mathrm{g}$. a day. With doses of $400^{\circ} \mu \mathrm{g}$. or more the drug was dissolved in half a pint ( $285 \mathrm{ml}$.) of saline, and given by intravenous drip over about two to three hours. A total course of between 7,000 and $10,000 \mu \mathrm{g}$. was used whenever possible. In one patient who responded to the drug but relapsed rapidly an attempt was made to maintain improvement with twice-weekly injections of $400 \mu \mathrm{g}$. This was carried out for two months, but did not appear to be effective.

\section{Results}

The results of treatment are summarized in Table I. Only two patients showed a marked response to treatment, with disappearance of fever and considerable reduction in the 